GUIMARÃES, D.F. et al. Estudo morfométrico do coração de avestruzes (Struthio camelus). PUBVET, Londrina, V. 5, N. 11, Ed. 158, Art. 1066, 2011.

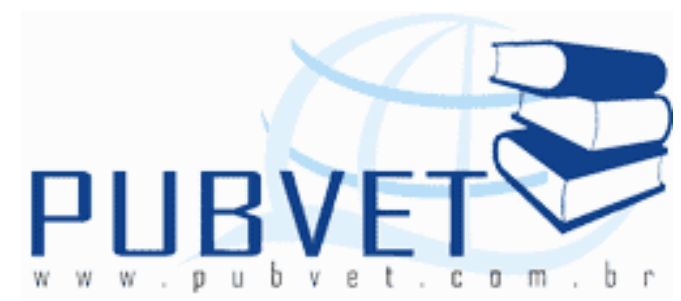

PUBVET, Publicações em Medicina Veterinária e Zootecnia.

\title{
Estudo morfométrico do coração de avestruzes (Struthio camelus)
}

Daniel Faustino Guimarães ${ }^{1}$ Ana Bárbara Freitas Rodrigues ${ }^{2}$; Leonardo Serafim da Silveira ${ }^{2}$ Alexandre Thomé da Silva Almeida ${ }^{3}$; Gabriel Barbosa Botelho de Souza ${ }^{1}$; Maurício Portes Santos Sobrado Silva ${ }^{1}$

${ }^{1}$ Discentes do curso de Medicina Veterinária da Universidade Estadual do Norte Fluminense Darcy Ribeiro (UENF), Laboratório de Morfologia e Patologia Animal (LMPA); danielfaustino@hotmail.com.

${ }^{2}$ Docentes do LMPA/UENF.

${ }^{3}$ Docente do Curso de Medicina Veterinária da Faculdade São Lucas - Porto Velho - RO.

\section{Resumo}

O aumento da estrutiocultura no Brasil ocorreu em função da legislação vigente, a Portaria $\mathrm{n}^{0} 36$ de 15 de março de 2002, onde o Ministério da Agricultura, Pesca e Abastecimento (MAPA) classifica o avestruz no contexto de avicultura industrial, não sendo mais considerada, uma ave exótica, mas sim doméstica. Os avestruzes estão sujeitos a muitas enfermidades que também acometem as aves domésticas. O conhecimento científico acerca das doenças de avestruzes é incompleto e fragmentado, e detalhes específicos sobre aspectos de diagnóstico 
GUIMARÃES, D.F. et al. Estudo morfométrico do coração de avestruzes (Struthio camelus). PUBVET, Londrina, V. 5, N. 11, Ed. 158, Art. 1066, 2011.

de doenças, dentre outros tópicos, são completamente inexistentes na maioria dos casos Diante deste contexto, este estudo teve a intenção de avaliar a morfometria cardíaca de seis avestruzes através da mensuração dos perímetros: das valvas atrioventriculares esquerda e direita, assim como o da valva aórtica e da valva pulmonar, e o do sulco coronário. Também foram mensuradas a espessura do septo interventricular, espessura da parede de ventrículos direitos e esquerdo, e a distância do sulco coronário ao ápice cardíaco. Os dados morfométricos obtidos tornam-se relevantes devido à carência de dados na literatura a respeito da anatomia cardíaca de avestruzes.

Palavras-Chave: Avestruz; coração; morfometria.

\title{
Morphometric study of the heart of ostrich (Struthio camelus)
}

\begin{abstract}
The increase of ostrich in Brazil was due to current legislation, Ordinance 36, n0 15 March 2002, where the Ministry of Agriculture, Fisheries and Food Supply (MAPA) ranks in the context of ostrich poultry industry, not being considered, an exotic bird, but domestic. Ostriches are subject to many diseases that also affect poultry. Scientific knowledge about diseases of ostriches is incomplete and fragmented, and specific details on aspects of disease diagnosis, among other topics, is completely absent in most cases. Given this context, this study intended to evaluate the cardiac morphology of six ostriches by measuring the perimeters: the left and right atrioventricular valves, as well as the aortic valve and the pulmonary valve and the coronary. Were also measured the thickness of the ventricular wall thickness of left and right ventricles, and the distance to the apex of the coronary heart disease. The morphometric data become relevant due to lack of data in the literature regarding cardiac anatomy of owls
\end{abstract}

Keywords: Ostrich; heart; morphometry 
GUIMARÃES, D.F. et al. Estudo morfométrico do coração de avestruzes (Struthio camelus). PUBVET, Londrina, V. 5, N. 11, Ed. 158, Art. 1066, 2011.

\section{INTRODUÇÃO}

O avestruz (Struthio camelus), animal pertencente ao grupo das ratitas, aves que não voam, tem ganhado espaço como importante alternativa no setor agropecuário, tendo em vista seu grande potencial para exploração racional como fonte de alguns produtos: carne, couro, plumas, dentre outros ${ }^{1,2}$ O País conta com cerca de 2.500 mil criadores distribuídos em todo o território nacional, com destaque para o estado de São Paulo, que detém em torno de 700 criadores e aproximadamente 120.000 avestruzes $^{3}$.

Os avestruzes estão sujeitos a muitas enfermidades que acometem as aves domésticas, com variações quanto à incidência das mesmas, susceptibilidade e resistência dos animais ${ }^{1}$. O conhecimento científico acerca das doenças de avestruzes é incompleto e fragmentado, sendo que detalhes específicos sobre aspectos de diagnóstico de doenças, dentre outros tópicos, são completamente inexistentes na maioria dos casos ${ }^{4}$.

Comparando os avestruzes com aves menores, que são capazes de voar, podemos perceber que eles têm uma grande tolerância ao estresse térmico na terra. Isto é exacerbado pelo fato que esses animais são muito grandes para procurarem abrigo e se protegerem com facilidade em ambientes com microclimas hostis ${ }^{5}$.

É fato que os avanços na área médica estão intrinsecamente relacionados com o progresso da pesquisa anatômica'. A anatomia não é um tópico morto. "É parte viva da constante pesquisa científica e também a base para todo 0 conhecimento biológico"7 . A função primordial da anatomia é determinar as estruturas do organismo, não só sobre o ponto de vista morfológico, mas também sob o ponto de vista de um organismo composto de diversos sistemas integrados entre si. O entendimento, por exemplo, de todos os mecanismos que envolvem o trabalho cardíaco bem como as estruturas que participam deste devem ser considerados $^{8}$. Para isto, estão disponíveis diversas técnicas de estudo, como a 
GUIMARÃES, D.F. et al. Estudo morfométrico do coração de avestruzes (Struthio camelus). PUBVET, Londrina, V. 5, N. 11, Ed. 158, Art. 1066, 2011.

morfometria, que tem servido de pilar de sustentação para o embasamento conceitual da anatomia e, em especial, da anatomia funcional, uma vez que os dados métricos de alguma forma refletem também a disposição funcional ${ }^{9}$.

De uma maneira geral, o coração das aves tem quatro câmaras e é semelhante ao dos mamíferos, exceto por algumas características secundárias ${ }^{10}$. De acordo com alguns estudos, o coração de avestruz é relativamente maior e sua freqüência de contração é muito mais alta,especialmente em animais jovens ${ }^{11}$.Em avestruzes, o coração possui uma forma cônica, com o ápice formado apenas pelo ventrículo esquerdo, estando situado na superfície côncava do esterno, o qual se encontra voltado caudalmente, com eixo longo perpendicular à parede ventral do corpo ${ }^{12}$.

O coração fica dentro do tórax, tanto entre os lobos hepáticos, como cranial a eles. É ligado ao esterno pelo pericárdio, o qual nas aves em geral, é um saco fibroso, consistente e translúcido, afixado às estruturas circundantes. O pericárdio envolve externamente o coração e contém espessa camada de tecido adiposo sobre o pericárdio fibroso e sob a lâmina visceral do pericárdio seroso (epicárdio), ocultando os ramos das artérias coronárias, principalmente no seio coronário ${ }^{13,14}$.

O coração da avestruz possui quatro câmaras, dois átrios e dois ventrículos. No átrio direito encontra-se a abertura das veias cava cranial e caudal, que desembocam nesta câmara. No átrio esquerdo há a desembocadura das veias pulmonares ${ }^{14}$. A valva atrioventricular direita é formada por uma única aba muscular desprovida de cordas tendíneas. O ventrículo direito, de paredes finas, situa-se ao redor do ventrículo esquerdo ${ }^{10}$. A parede do ventrículo esquerdo é consideravelmente mais espessa que a do ventrículo direito e é possível observar as trabéculas carnosas em sua parede interna ${ }^{14}$. O ventrículo esquerdo, de paredes espessas, tem a forma de um cilindro pontiagudo. Barras musculares em seu interior dão, ao corte transverso, um aspecto de roseta ${ }^{10}$. Em geral, o miocárdio ventricular esquerdo das aves é bastante espesso. Em sua maior parte, 
GUIMARÃES, D.F. et al. Estudo morfométrico do coração de avestruzes (Struthio camelus). PUBVET, Londrina, V. 5, N. 11, Ed. 158, Art. 1066, 2011.

a parede ventricular esquerda é de três a quatro vezes mais espessa do que a do ventrículo direito ${ }^{13}$, como demonstrado na figura 1 e 2 .

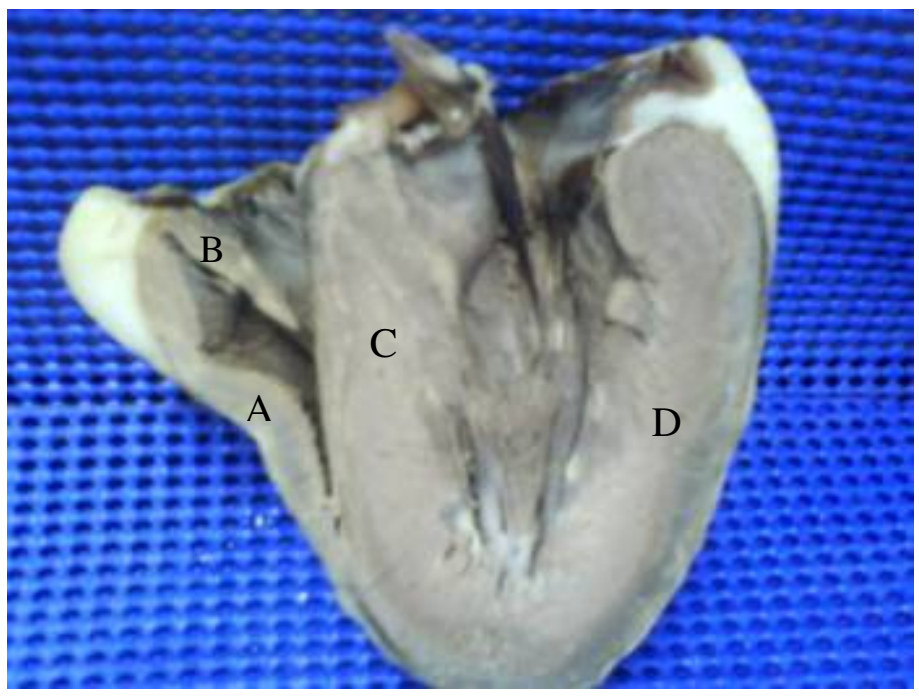

Figura 1: Corte Longitudinal do coração de avestruz; A- Parede do ventrículo direito; B- Lâmina muscular; C- Septo Interventricular; D- Parede do ventrículo esquerdo.

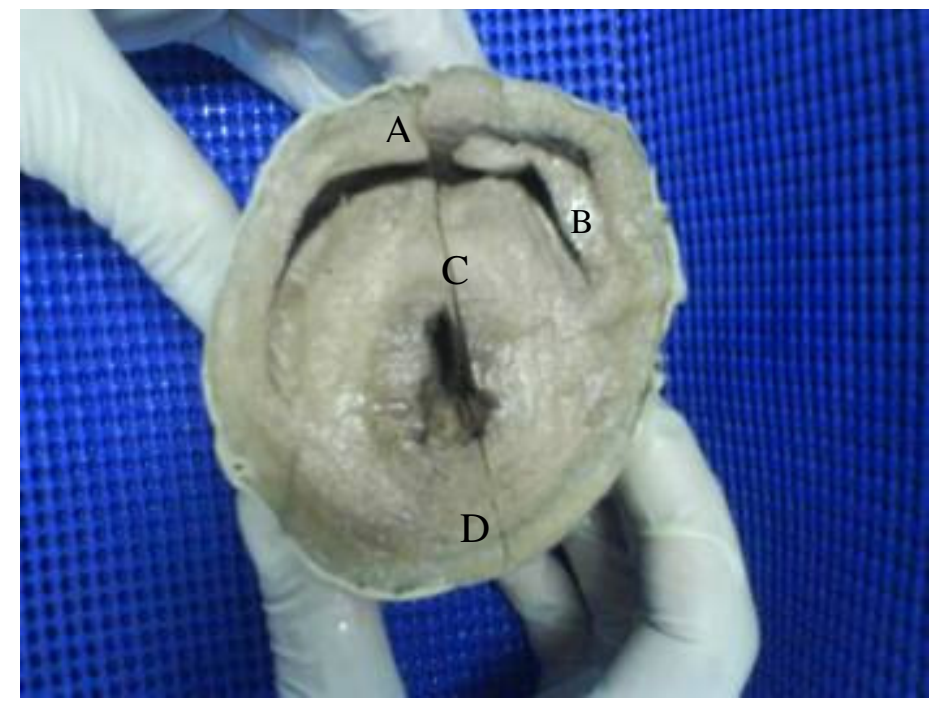

Figura 2: Corte Transversal do coração de avestruz; A- Parede do ventrículo direito; B- Lâmina muscular; C- Septo Interventricular; D- Parede do ventrículo esquerdo. 
GUIMARÃES, D.F. et al. Estudo morfométrico do coração de avestruzes (Struthio camelus). PUBVET, Londrina, V. 5, N. 11, Ed. 158, Art. 1066, 2011.

Ao estudarem as valvas cardíacas de avestruzes, foi observado que a valva atrioventricular direita apresenta apenas uma lâmina muscular com duas fixações, já a esquerda se apresenta com três válvulas, uma dorsal, uma esquerda e uma direita ou septal ${ }^{10}$. No tronco pulmonar e na aorta é possível identificar as valvas semilunares, pulmonar e aórtica, respectivamente. Essas valvas são amplas, profundas e elásticas ${ }^{14}$. O segmento ligeiramente bulboso produzido pelos três seios pulmonares está localizado na base do curto tronco pulmonar. Cada seio pulmonar contém uma cúspide semilunar da valva pulmonar que evita o refluxo do sangue do tronco pulmonar para o cone. O bulbo da aorta consiste em três expansões bulbosas distintas, os seios da aorta, para dentro dos quais as cúspides da valva aórtica achatam-se durante a sístole ventricular ${ }^{13}$, como mostra a figura 3.

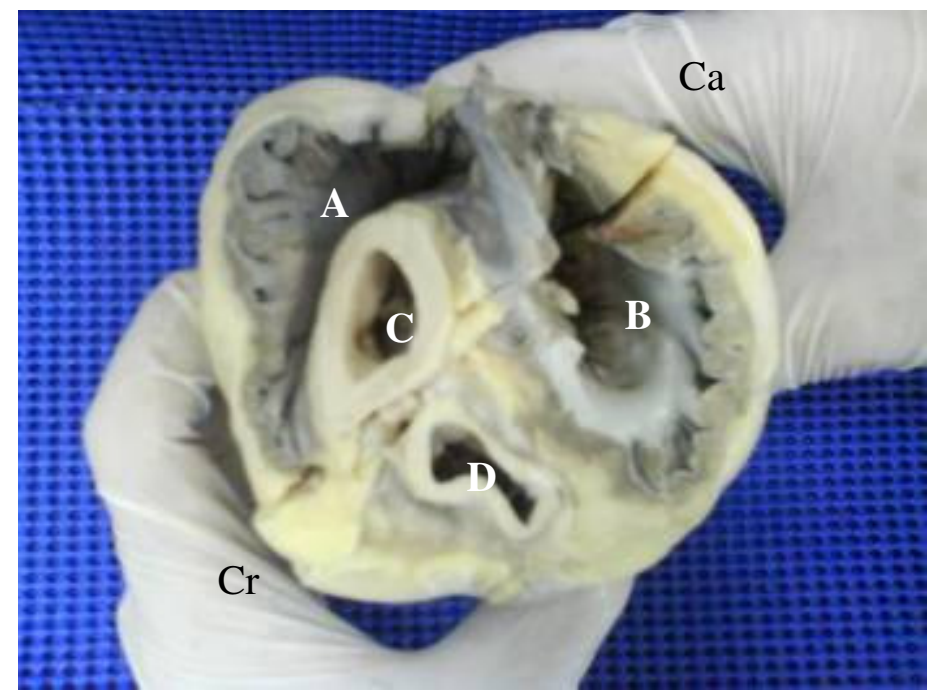

Figura 3: Corte Transversal do coração de avestruz, na altura das valvas cardíacas; Cr-Cranial; Ca- Caudal; A- Valva Atrioventricular direita; B- Valva Atrioventricular esquerda; C- Valva Aórtica ; D- Valva Pulmonar. 
GUIMARÃES, D.F. et al. Estudo morfométrico do coração de avestruzes (Struthio camelus). PUBVET, Londrina, V. 5, N. 11, Ed. 158, Art. 1066, 2011.

Atualmente, é incontestável a importância que adquiriu a cardiologia, juntamente com os avanços científicos que vêm ocorrendo de forma constante, e torna-se evidente a necessidade de realização de estudos que objetivem conhecer as características presentes nas estruturas anatômicas cardíacas dos animais ${ }^{6}$. Como o trabalho muscular tem grande demanda de nutrientes e oxigênio, a avestruz necessita de um sistema cardiovascular que corresponda a essa atividade. Com isso, descrições sobre a morfologia do coração tornam-se importantes para o desenvolvimento da exploração comercial dessa ave $\mathrm{e}^{14}$.

Esse trabalho teve como propósito determinar os padrões morfométricos do coração de avestruz utilizando como ferramenta básica o estudo macroscópico para definir as características estruturais do coração desta espécie. Sabe-se que o êxito técnico do ato operatório está intimamente ligado a um profundo conhecimento da anatomia, conseguindo-se com isso uma melhor compreensão das patologias que porventura possam a ocorrer.

\section{MATERIAL E MÉTODOS}

Para desenvolvimento do presente trabalho foram utilizados seis corações de avestruzes, machos e fêmeas, com idade entre 12 e 18 meses, pesando em média $75 \mathrm{Kg}$. Os corações estudados foram coletados. durante o abate, na linha de inspeção, de um frigorífico sob inspeção estadual. Após serem retirados da cavidade celomática, os corações foram previamente lavados, com água corrente, para a retirada do sangue restante nas câmaras cardíacas, e em seguida identificados e acondicionados em refrigeração até serem levados para o setor de Anatomia da Universidade Estadual do Norte Fluminense Darcy Ribeiro, onde as peças anatômicas foram processadas individualmente. Foi retirada de 
GUIMARÃES, D.F. et al. Estudo morfométrico do coração de avestruzes (Struthio camelus). PUBVET, Londrina, V. 5, N. 11, Ed. 158, Art. 1066, 2011.

cada peça a gordura excessiva da base dos grandes vasos e efetuadas as medidas referentes ao perímetro maior do coração (na altura do sulco coronário) e a distância da base até o ápice. Em seguida, os corações foram imersos em uma solução de formol tamponado $10 \%$ por um período de 15 dias para a perfeita fixação, segundo protocolo proposto ${ }^{15}$. Ao término deste período, os corações foram seccionados longitudinalmente, no sentido base-ápice, para que as cavidades ventriculares e atriais fossem expostas. A partir da exposição das câmaras cardíacas, e tendo como base o terço médio da face esquerda de cada ventrículo, foram tomadas medidas referentes a espessura do septo interventricular, a espessura da parede ventricular direita e esquerda e a distância do sulco coronário ao ápice. Para proceder à mensuração da espessura do septo interventricular, da espessura da parede ventricular direita e esquerda, e a distância do sulco coronário ao ápice, foi utilizado um paquímetro Mitotoyo. $O$ perímetro maior do coração (na altura do sulco coronário) foi mensurado com o auxílio de um fio de sutura, que foi colocado em torno da referida área para que a mesma fosse moldada e posteriormente este fio foi transposto para uma régua, fornecendo então a medida de interesse. Dessa mesma forma, os perímetros das quatro valvas cardíacas foram mensurados.

\section{RESULTADO E DISCUSSÃO}

Diante de uma avaliação macroscópica dos corações estudados foi possível constatar o formato cônico e as características estruturas valvares compatíveis com as já descritas ${ }^{12,14}$. No presente estudo, pudemos constatar que dados referentes a morfologia interna e externa do coração de avestruzes são semelhantes às descritas em outras aves domésticas como a galinhas ${ }^{10}$.

A morfologia das valvas atrioventriculares esquerdas analisadas foram semelhante às descrições já existentes ${ }^{10}$ e apresentaram uma média aritmética 
GUIMARÃES, D.F. et al. Estudo morfométrico do coração de avestruzes (Struthio camelus). PUBVET, Londrina, V. 5, N. 11, Ed. 158, Art. 1066, 2011.

dos perímetros de $9,025 \mathrm{~cm}$. As valvas atrioventriculares direitas estavam disposta em uma lâmina muscular grossa, assim como nas galinhas e outros pássaros ${ }^{13}$ e a média aritmética de seus perímetros foi de 9,862 cm. Em relação às valvas aórtica e pulmonar estas apresentaram médias aritméticas dos seus perímetros de $7,787 \mathrm{~cm}$ e $7,237 \mathrm{~cm}$, respectivamente e características morfológicas que corrobam com as já descritas ${ }^{14}$.

Através da análise morfológica dos corações analisados foi identificar um ventrículo direito de paredes finas e situado ao redor do ventrículo esquerdo, de forma que seu lúmen, no corte transverso, tem forma de crescente ${ }^{10}$. Já o ventriculo esquerdo apresentou um miocárdio ventricular bastante espesso,evidenciando uma parede ventricular três a quatro vezes mais espessa do que a do ventrículo direito ${ }^{13}$. Os valores médios obtidos nas mensurações dos corações estudados foram os seguintes: perímetro do sulco coronário $(28.9 \mathrm{~cm})$, distância da base ao ápice $(14,7 \mathrm{~cm})$, espessura do septo interventricular $(2,24 \mathrm{~cm})$, espessura da parede ventricular direita $(1,17 \mathrm{~cm})$, espessura da parede ventricular esquerda $(2,25)$ e a distância do sulco coronário ao ápice $(9,18 \mathrm{~cm})$.

As informações disponíveis na literatura em relação a anatomia cardíaca de avestruzes são escassas, predominando estudos relativos à anatomia cardíaca de animais domésticos como cães, eqüino e suínos ${ }^{13}$. Ao realizarmos este estudo morfométrico em avestruz, buscamos adquirir dados específicos para uma padronização da anatomia cardíaca destes animais, já que existe uma carência de informações referente a morfometria cardíaca desta espécie. Apesar da crescente evolução da estrutiocultura no Brasil ainda é muito incipiente o conhecimento científico em relação a anatomofisiologia dos avestruzes. Ao tentarmos discutir os resultados encontrados no presente estudo com os dados disponíveis na literatura encontramos grandes dificuldades, reconhecendo desta maneira que pouca 
GUIMARÃES, D.F. et al. Estudo morfométrico do coração de avestruzes (Struthio camelus). PUBVET, Londrina, V. 5, N. 11, Ed. 158, Art. 1066, 2011.

atenção tem sido direcionada para um maior conhecimento dos aspectos morfológicos e morfométricos do coração de avestruz.

\section{REFERÊNCIAS BIBLIOGRÁFICAS}

1.CARRER, C.C., KORNFELD, M.E. A criação de avestruzes no Brasil. Pirassununga: Brasil Ostrich Comercial., 1999, 304p.

2.HUCHZERMEYER, F. W. Doenças de avestruzes e outras ratitas. 2aedição. Jaboticabal: Funep, 2000, 392p.

3. ACAB (2006) Comunicado oficial da Associação dos Criadores de Avestruz do Brasil. http://www.acab.org.br em 01/02/2008.

4.VERWOERD, D.J. Ostrich disease. Reviews in Science Technology. v.19, n.2, p. 638-661, 2000.

5.MAINA, J. N.; NATHANIEL, C. A qualitative and quantitative study of the lung of an ostrich, Struthio Camelus. The Journal of Experimental Biology. v.204, p. 2313-2330,2001.

6.POGLIANI, F.C.; STOPIGLIA, A. J.; MARIANA, A. N. B.; SPERS, A.; MIGLIANO, M. A.; FREITAS, R. R.; IRINO, E. T.; FERREIRA, F.; JATENE, F. B.; DI DIO, L. J. A. Morfometria cardíaca em coelhos (oryctolagus cuniculus), da raça Nova Zelândia. Res. Brás. Med. Vet. v.25, n.5/6, p.215-218,2003.

7.HERRICK, J. B.In : SISSON \& GROSSMAM. Anatomia dos Animais Domésticos. 5a Ed. Rio de Janeiro, Editora Guanabara Koogan. 1986, p.xxiii.

8.BENEDICTO,H.G.;BOMBONATO, P.P. Brazilian Journal of Veterinary Reserch and Animal Science. 40: 108-116,2003.

9.FREITAS, R. R.; STOPIGLIA, A. J.; JATENE, F.B.; MACRUZ, R.; ARMIGLIATO, A. L.; ANELI, E.; MONTEIRO, R. Estudos comparativos das relações entre a aorta e a artéria tronco pulmonar. vet. Not. Uberlândia. v.8, n.1, p.11-16, 2002.

10.DYCE,K.M.; SACK,W.O.;WENSING,C.J.G.. Tratado de Anatomia Veterinária, Rio de Janeiro:Elsevier, 2004, p.773-779.

11. REZAKHANI, A., KOMALI, H., MOKHBERDEZFOUL, M. R., ZARIFI, M., GHABI, M., ALIDADI, N., NADALIAN, M. G. A preliminary study on normal electrocardiographic parameters of ostriches (Struthio camelus). Journal of the South African Veterinary Association. v. 78, n. 1, p. 46-48, 2007.

12.BEZUIDENHOUT, A. J. The topography of the thoraco-abdominal viscera in the ostrich (Struthio camelus). Onderstepoort Journal of Veterinary Research. v. 53, n. 2, p. 111-117, 1986.

13. BAUMEL, J. J. Coração e vasos sangüíneos das aves.In: Getty, R. (Ed.). Anatomia dos animais domésticos. 5a ed. Rio de Janeiro: Guanabara Koogan,1986. p.1842-1880.

14. SOARES, G.L., OLIVEIRA,D., BARALDI-ARTONI, S. M. Aspectos da anatomia do coração do avestruz. Ars Veterinaria, Jaboticabal, SP, v.26, n.1, p. 38-42, 2010.

15. RODRIGUES, H.Técnicas Anatômicas. 2a ed. Vitória, 1932, 222p. 